\title{
Rate, Sub-Carrier, and Power Allocations for Multi-Carrier CDMA with LMMSE Multiuser Detection
}

\author{
Po-Wei Fu, Member, IEEE, and Kwang-Cheng Chen, Senior Member, IEEE
}

\begin{abstract}
Multi-Carrier Code-Division-Multiple-Access (MCCDMA) is a promising transmission technique for high-speed wireless multimedia communication in frequency-selective fading channels. In this letter, allocations of physical transmission rate, sub-carrier, and power are proposed for a MC-CDMA system that applies Multi-Code (MC)/Variable-Spreading-Length (VSL) multi-rate access to minimize total transmitted power, where we consider users have different data rate and BER requirements and LMMSE multiuser detection is used in the receivers. We derive transmission rate capacity and propose a simple admission control criterion that linearly relates the code length, data rate and BER requests of all users. The proposed iterative allocation algorithm jointly allocates the sub-carrier power of each user and solves the sub-carrier allocation problem.
\end{abstract}

Index Terms-Multi-Carrier CDMA, power allocation, subcarrier allocation, multi-code, variable-spreading-length

\section{INTRODUCTION}

W IRELESS multimedia communications are playing increasingly important roles in the emerging communication generations to serve various applications, which require not only effective transmission technique but also resource allocation to provide different quality-of-services (QoS) for users of various demands. MC-CDMA that combines Orthogonal-Frequency-Division-Multiplexing (OFDM) and CDMA was shown its advantages in transmitting broadband signals over frequency-selective fading channels and providing high capacity [1], [2]. It is regarded as a promising candidate to replace single-carrier DS-CDMA for advanced multimedia applications in systems beyond 3G. In addition to effective receiver design, system optimization by efficient resource allocation is then a critical topic for wireless multimedia communications.

Data stream in MC-CDMA is spread by a given spreading code and each code chip modulates a different sub-carrier, which is the so-called frequency-domain spread spectrum [1][3] and all sub-carriers are mutually orthogonal. Power, subcarrier, and spreading codes are available radio resources in MC-CDMA. Conventionally, power is uniformly distributed to all sub-carriers without optimization and it is obviously inefficient according to the information theory. Providing channel state information to the transmitters, a method ever proposed to turn off deep-faded sub-carriers and uniformly distribute power to the remaining sub-carriers to improve the performance of maximum-ratio-combining (MRC) receiver

Manuscript received February 1, 2005; revised July 1, 2006; accepted September 20, 2006. The associate editor coordinating the review of this letter and approving it for publication was A. Scaglione.

The authors are with the Graduate Institute of Communication Engineering, National Taiwan University, No. 1, Sec. 4, Roosevelt Rood., BL-504,Taipei, Taiwan, 10617 (e-mail: \{powei, chenck \}@ee.ntu.edu.tw).

Digital Object Identifier 10.1109/TWC.2007.05022.
[4]. However, it is not proven an optimal method in any sense. Most previous research about resource allocation in CDMA systems only considered conventional matched-filterbased single-user-detection (SUD) receivers [5]-[7], where simplified signal-to-interference-ratio (SINR)-based formulations were used to treat the interference as merely an accumulated power sum of all users, but it is well- known that multiuser detection (MUD) can significantly improve the performance and capacity of CDMA systems [8] and especially recommended in MC-CDMA uplink applications due to the serious distortion of code orthogonality in frequencyselective fading channels [9]. Hence, radio resource allocation with MUD receivers is essential to practical MC-CDMA applications.

For multi-rate transmissions, Multi-Code (MC) and Variable-Spreading-Length (VSL) are two widely adopted multi-rate schemes in CDMA systems [10], and their applications in MC-CDMA were studied [11] with LMMSE MUD effectively to mitigate multiple access interference and orthogonality distortions. In this letter, we consider all users in a MC-CDMA system have individual QoS demands on data rate and bit-error-rate (BER), and we allocate transmission rate, sub-carriers, and sub-carrier power to minimize total transmitted power. For easily dealing with the nature of MC/VSL access, the optimization is processed over the domain where users are decomposed as unit-rate virtual users such that these two kinds of multi-rate MC-CDMA systems exhibits structural regularity. We derive the user capacity under their QoS constraints and propose a simple but practical user (or data stream) admission criterion. To allocate power of each admitted user, an iterative allocation algorithm is proposed to adjust sub-carrier power and phase, in which sub-carrier selection is jointly achieved.

\section{SySTEM DESCRIPTION AND LMMSE MUD}

\section{A. MC MC-CDMA \& VSL MC-CDMA}

Suppose the data rates of all users are integer multiples of a pre-defined basic rate $R_{b}$, where $R_{b}=T_{s}^{-1}$ and define $F$ as the basic spreading length, i.e., the code length of basic-rate users in the system. A "rate- $m$ " user means a user whose data rate is $m R_{b}$. In MC-CDMA, every symbol is duplicated to several copies and each is multiplied by a chip of the given spreading code, where these modulated copies are transmitted in parallel by orthogonal sub-carriers [1], [2]. For a rate- $m$ user, MC MC-CDMA multiplexes the source data stream into $m$ basic-rate data streams and these data streams are transmitted respectively with different spreading codes by MC-CDMA transmission procedure. Instead of spreading code multiplexing, VSL MC-CDMA adjusts the number of 


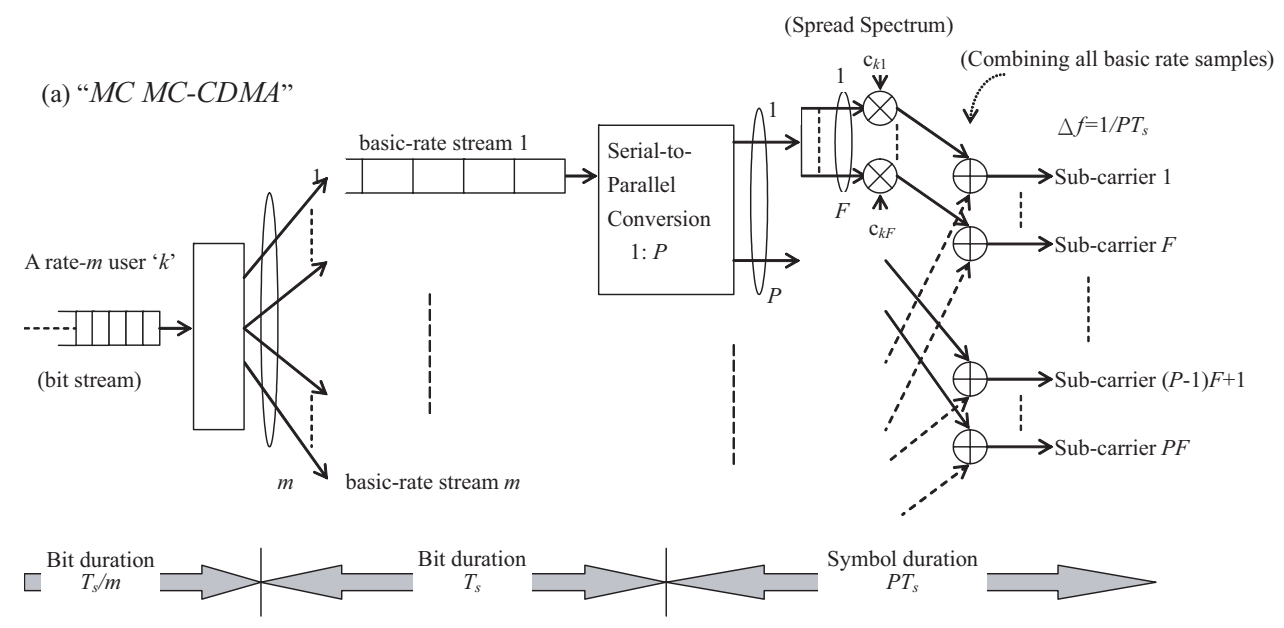

(b) "VSL MC-CDMA"

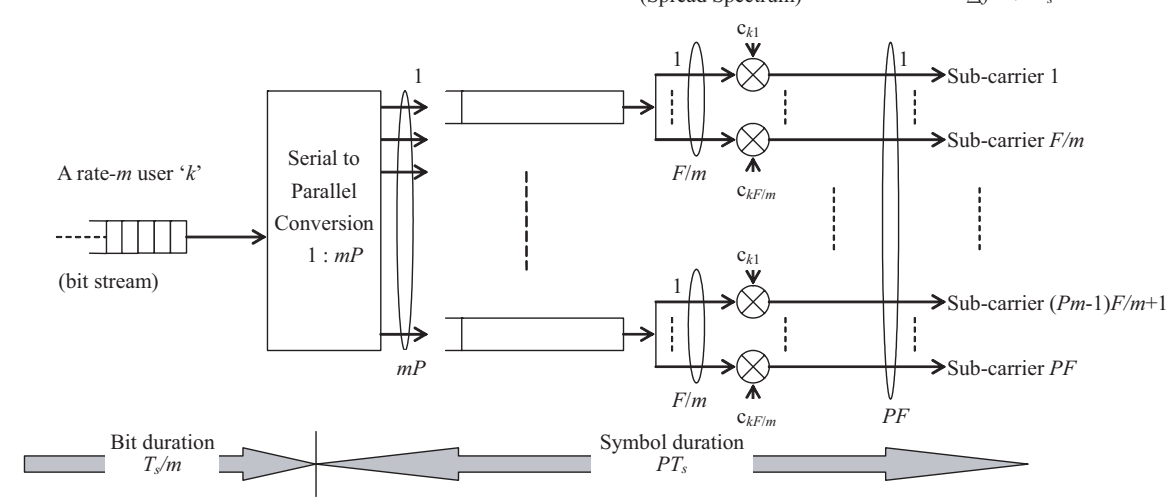

Fig. 1. MC MC-CDMA and VSL MC-CDMA transmissions.

used sub-carriers in the MC-CDMA transmission procedure to fit various data rates, where $F / m$, an integer, sub-carriers are used for each symbol of a rate- $m$ user. Fig. 1 illustrates MC/VSL MC-CDMA transmissions.

To simplify the analysis, a rate- $m$ user in either $\mathrm{MC}$ or VSL MC-CDMA system can be decomposed as $m$ basic-rate virtual users (like the effective users in single-carrier multi-rate CDMA [10]), where all virtual users have identical symbol durations and the lengths of spreading codes $F$ [11]. For example of a system whose basic spreading length is 4 , a double-rate user can be decomposed as two virtual users with spreading code [ $\left[\begin{array}{llll}1 & -1 & 1 & -1\end{array}\right]$ and $\left[\begin{array}{llll}1 & 1 & -1 & -1\end{array}\right]$ in MC access, and with spreading codes $\left[\begin{array}{llll}1 & -1 & 0 & 0\end{array}\right]$ and [ $\left[\begin{array}{llll}0 & 0 & 1 & -1\end{array}\right]$ in VSL access. If $K_{m}$ denotes the number of active rate- $m$ users, where $m=1,2, \ldots, M$, the number of total virtual users is $K=\sum_{m=1}^{M} m K_{m}$. The received signal in the receiver can be modeled for both access schemes as:

$r(t)=\sum_{k=1}^{K} A_{k f} b_{k} \sum_{f=1}^{F} \alpha_{k f} c_{k f} e^{j 2 \pi(f-1) \Delta f}+n(t), 0 \leq t \leq T_{s}$,

where $A_{k f}$ is the transmitted amplitude of the virtual user $k$ at the sub-carrier $f, b_{k}$ denotes the source symbol of the virtual user $k$ within an OFDM symbol period, $\alpha_{k f}$ denotes the coefficient of the sub-channel passed by the $f$ th chip of $b_{k}$, and $n(t)$ is the additive white Gaussian noise (AWGN) with power spectrum density $N_{0} / 2$. We assume the number of total sub-carriers is large enough to yield flat fading in every subchannel, which is an essential operation requirement for MCCDMA [1]-[3], and assume the fading is slow with respect to the OFDM symbol period, $T_{s}$. We model BPSK modulation here for simplicity, and $b_{k}$ can be a complex variable in QAMfamily modulations.

\section{B. LMMSE Multiuser Detection}

After down-conversion to baseband, the received samples from all sub-carriers compose a $F \times 1$ vector $\mathbf{r}$, where

$$
\begin{aligned}
\mathbf{r} & =\left[\begin{array}{c}
r_{1} \\
r_{2} \\
\vdots \\
r_{F}
\end{array}\right] \\
= & \underbrace{\left[\begin{array}{ccc}
A_{11} \alpha_{11} c_{11} & \ldots & A_{K 1} \alpha_{K 1} c_{K 1} \\
A_{12} \alpha_{12} c_{12} & \ldots & A_{K 2} \alpha_{K 2} c_{K 2} \\
\vdots & \ddots & \vdots \\
A_{1 F} \alpha_{1 F} c_{1 F} & \ldots & A_{K F} \alpha_{K F} c_{K F}
\end{array}\right]}_{\mathbf{M}} \underbrace{\left[\begin{array}{c}
b_{1} \\
b_{2} \\
\vdots \\
b_{K}
\end{array}\right]}_{\mathbf{b}}+\underbrace{\left[\begin{array}{c}
n_{1} \\
n_{2} \\
\vdots \\
n_{F}
\end{array}\right]}_{\mathbf{n}}
\end{aligned}
$$


with $n_{f}=\int_{0}^{P T_{s}} n(t) \exp \left(-j 2 \pi T_{s}\right) d t$. Denote $\mathbf{W}$ as the linear filter operating over $\mathbf{r}$ that minimizes the mean-squared-error (MSE) $E\left[|\mathbf{W r}-\mathbf{b}|^{2}\right]$, and the LMMSE MUD is [11]

$$
\left\{\begin{array}{l}
\hat{\mathbf{b}}=\operatorname{sign}(\operatorname{Re}\{\mathbf{W r}\}) \\
\mathbf{W}=\mathbf{M}^{\mathrm{H}}\left(\mathbf{M M}^{\mathrm{H}}+\frac{N_{0}}{2} \mathbf{I}\right)^{-1}
\end{array}\right.
$$

where $\hat{\mathbf{b}}$ is the decisions for $\mathbf{b}$ and the achieved MSE for virtual user $k$ is:

$$
\mathrm{MSE}_{k}=1-\mathbf{m}_{k}^{\mathrm{H}}\left(\sum_{j=1}^{K} \mathbf{m}_{j} \mathbf{m}_{j}^{\mathrm{H}}+\frac{N_{0}}{2} \mathbf{I}\right)^{-1} \mathbf{m}_{k},
$$

and $\mathbf{m}_{k}$ is the $k$ th column of the matrix $\mathbf{M}$.

The MUD in (3) is identically applicable to both MC MC-CDMA and VSL MC-CDMA. By Gaussian approximating [12] the interference from plenty of virtual users, the error probability with respect to the bit of a virtual user $P_{e}=Q(\sqrt{\mathrm{SINR}})$, where the SINR (signal-to-interferencewith-noise power ratio) can be proved, by following its similarity to the LMMSE MUD in DS-CDMA [13], that

$$
\mathrm{SINR}_{k}=\frac{1}{\mathrm{MSE}_{k}}-1, \forall k
$$

Actually, it can be mathematically proved that the filter $\mathbf{W}$ operated on $\mathbf{r}$ is equivalent to the filter $\tilde{\mathbf{W}}$ operated on $\mathbf{y}$, where

$$
\tilde{\mathbf{W}}=\left(\mathbf{R}+\frac{N_{0}}{2} \mathbf{I}\right)^{-1},
$$

$\mathbf{R}=\mathbf{M}^{\mathrm{H}} \mathbf{M}$, and $\mathbf{y}=\mathbf{M}^{\mathrm{H}} \mathbf{r}$. The achieved MSE for the $k$ th virtual user has the equivalent form:

$$
\mathrm{MSE}_{k}=1-\mathbf{r}_{k}^{\mathrm{H}}\left(\sum_{j=1}^{K} \mathbf{r}_{j} \mathbf{r}_{j}^{\mathrm{H}}+\frac{N_{0}}{2} \mathbf{R}\right)^{-1} \mathbf{r}_{k},
$$

where $\mathbf{r}_{k}$ denotes the $k$ th column of $\mathbf{R}$. Such alternative avoids the numerical singularity at high signal-to-noise ratio (SNR) environments when $F>K$. Hence, we consider the filter (6) in the receiver but the equivalent MSE form in (4) contributes to determining the user capacity and developing the admission criterion, shown in the next section.

\section{CAPACITY AND RATE Allocation}

Assume each user has individual QoS requirements on data rate and tolerable BER and denote the request of user $i$ as $\left(\hat{m}_{i}, \gamma_{i}\right)$ for its rate demand $\hat{R}_{i}=\hat{m}_{i} R_{b}$ and SINR target $\gamma_{i}$. Note that $\hat{m}_{i}$ is an integer. By the virtual user model, the demand of each user can be translated into the number of virtual users requested to be transmitted and these virtual users have the same SINR requirement in reception. Hence, if there are $N$ users requesting $\left(\hat{m}_{1}, \gamma_{1}\right), \ldots$, and $\left(\hat{m}_{N}, \gamma_{N}\right)$ in either MC MC-CDMA or VSL MC-CDMA system, to minimize total transmitted power for all users results in an optimization problem in terms of virtual users as:

$$
\text { Minimize } \quad \sum_{k=1}^{K} \sum_{f=1}^{F} A_{k f}^{2}
$$

Subject to: $\operatorname{SINR}_{k} \geq \gamma_{k}, k=1,2, \ldots, K$.

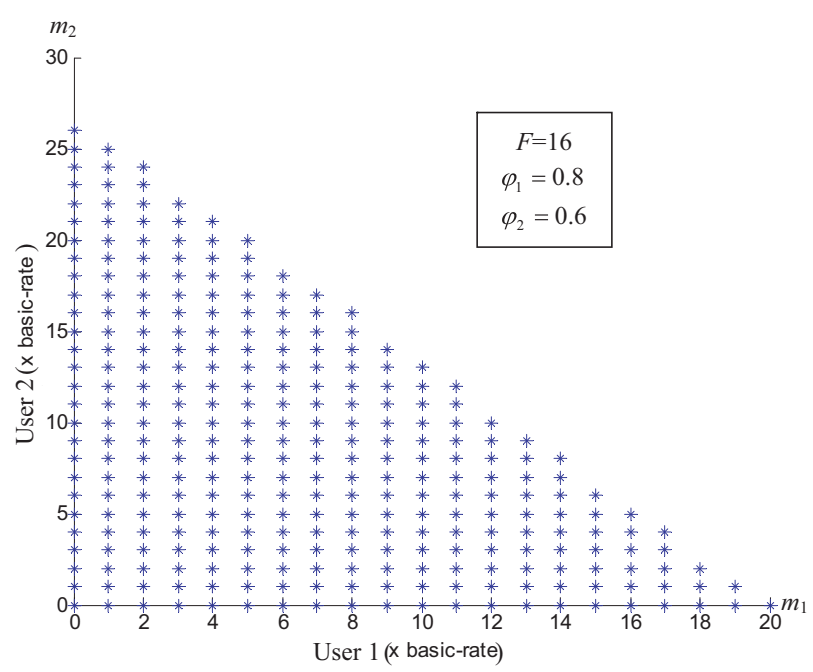

Fig. 2. The region of achievable transmission rates $\left(m_{1}, m_{2}\right)$ of a two-user example in MC-CDMA with LMMSE MUD. The MSE targets of User 1 and User 2 are $\varphi_{1}=0.8$ and $\varphi_{2}=0.6$ respectively.

$$
\begin{aligned}
m_{i} & \geq \hat{m}_{i}, i=1,2, \ldots, N . \\
K & =\sum_{i=1}^{N} m_{i}
\end{aligned}
$$

where $K$ is the number of virtual users corresponding to the requested rates of these $N$ users and $\gamma_{1}, \gamma_{2}, \cdots, \gamma_{K}$ are their SINR targets.

Proposition: " $\sum_{n=1}^{N} m_{n} \varphi_{n} \leq F$ " can be used as the admission criterion for the entrance of new user or new service, which meanwhile keeps guaranteeing the promised QoS.

In terms of virtual users, " $\sum_{n=1}^{N} m_{n} \varphi_{n} \leq F$ " is equivalent to " $F \geq \sum_{k=1}^{K} \varphi_{k}$ ". For mathematical convenience, replace the SINR constraints in (9) by the MSE constraints according to (5) as:

$$
\operatorname{MSE}_{k} \leq \frac{1}{\gamma_{k}+1}, k=1,2, \ldots, K .
$$

and let $\varphi_{k}=1-\left(\gamma_{k}+1\right)^{-1}$ as the equivalent MSE target of virtual user $k$, which follows by (4) that:

$$
\mathbf{m}_{k}^{\mathrm{H}}\left(\sum_{j=1}^{K} \mathbf{m}_{j} \mathbf{m}_{j}^{\mathrm{H}}+\frac{N_{0}}{2} \mathbf{I}\right)^{-1} \mathbf{m}_{k} \geq \varphi_{k}, k=1,2, \ldots, K .
$$

With such mathematical structure of (13), like the proof in [14], it can be shown that " $F \geq \sum_{k=1}^{K} \varphi_{k}$ " is the sufficient and necessary condition for feasible solutions. Denote the expected virtual users from the new user $j$ as virtual user $K+1, K+2$, $\ldots$, with MSE targets $\varphi_{K+1}, \varphi_{K+2}, \ldots$.respectively. We can hence decide the entrance of user $j$ by the following rule:

$$
\begin{cases}F \geq \sum_{k=1}^{K+\hat{m}_{j}} \varphi_{k}, & \text { admit user } j \\ F<\sum_{k=1}^{K+\hat{m}_{j}} \varphi_{k}, & \text { reject user } j\end{cases}
$$

Note that $\varphi_{K+1}=\varphi_{K+2}=\ldots=\varphi_{K+\hat{m}_{j}}$.

Actually, if $F \geq K$, there is always a feasible solution. Since there are more available sub-carriers than virtual users, 
we can always assign every virtual user a different subcarrier, regardless in MC MC-CDMA or VSL MC-CDMA, and the target SINR of each virtual user can be achieved given adequate power. Under such condition, the sub-carrier and power allocation result is similar to [14], although the system in [14] considered SUD and its multi-rate transmission was realized by changing code rate. Transmission by this way is essentially OFDMA (Orthogonal-Frequency-DivisionMultiple-Access, see [15]), which means OFDMA a special solution of (MC/VSL) MC-CDMA under sub-carrier/power allocations if " $F \geq K$ ".

This admission criterion also defines the capacity of MC/VSL MC-CDMA systems. The capacity region is a hyperlattice domain determined by the number of available subcarriers and the MSE targets of all requesting users. Fig. 2 plots the capacity region of a two-user example. These lattice points represent the supportable transmission rates. Such discrete rate is the feature resulting from $\mathrm{MC}$ and VSL multi-rate access. Although this criterion is identically applicable to MC MC-CDMA and VSL MC-CDMA, it should be noted that the feasible transmission rate of VSL access is a sub-set of the region shown in Fig. 2, where only the rates corresponding to power of 2 are available. This is because the length of VSL code is generally power of 2. Hence, VSL MCCDMA potentially can be further improved, by time-division tricks for example, to fully utilize the capacity at all lattice points.

\section{Sub-CARrier ANd Power Allocations}

To minimize total transmitted power, the granted users should transmit their signals according to their minimum requested rates, which results in $K=\sum_{i=1}^{N} \hat{m}_{i}$ for equations (8) to (11). By means of Lagrange multiplier method [16], we have the Lagrange equation:

$L=\sum_{k=1}^{K} \sum_{i=f}^{F} A_{k f}^{2}-\sum_{k=1}^{K} \lambda_{k}\left(\mathbf{r}_{k}^{\mathrm{H}}\left(\sum_{j=1}^{K} \mathbf{r}_{j} \mathbf{r}_{j}^{\mathrm{H}}+\frac{N_{0}}{2} \mathbf{R}\right)^{-1} \mathbf{r}_{k}-\varphi_{k}\right)$

where $\lambda_{k}$ is the multiplier corresponding to the MSE constraint of virtual user $k$. However, the complexity of the widely applied Steepest Descent method is highly complicated in practice, where the iterations for each user need the parameters regarding all other users. It prohibits the applications in fast varying channels and in the environments where user gets in and out frequently. Alternatively, reformulate the left-handside of (9),

$$
\mathbf{r}_{k}^{\mathrm{H}}\left(\sum_{j=1}^{K} \mathbf{r}_{j} \mathbf{r}_{j}^{\mathrm{H}}+\frac{N_{0}}{2} \mathbf{R}\right)^{-1} \mathbf{r}_{k}=\tilde{\mathbf{w}}_{k} \mathbf{M}^{H} \hat{\mathbf{C}}_{k} \mathbf{A}_{k}
$$

where $\tilde{\mathbf{w}}_{k}$ is the $k$ th row of $\tilde{\mathbf{W}}$, i.e., the filter coefficients for the $k$ th virtual user,

$$
\hat{\mathbf{C}}_{k}=\left[\begin{array}{cccc}
\alpha_{k 1} c_{k 1} & 0 & \ldots & 0 \\
0 & \alpha_{k 2} c_{k 2} & \ddots & \vdots \\
\vdots & \ddots & \ddots & 0 \\
0 & \ldots & 0 & \alpha_{k F} c_{k F}
\end{array}\right] \text { and } \mathbf{A}_{k}=\left[\begin{array}{c}
A_{k 1} \\
A_{k 2} \\
\vdots \\
A_{k F}
\end{array}\right]
$$

Hence, the MSE constraint becomes

$$
\tilde{\mathbf{w}}_{k} \mathbf{M}^{\mathrm{H}} \hat{\mathbf{C}}_{k} \mathbf{A}_{k} \geq \varphi_{k}, \forall k .
$$

This equation relates the transmitted power at every subcarrier, the filter coefficients in the receiver, and the MSE target of each virtual user. Let

$$
\mathbf{g}_{k}(n) \equiv \tilde{\mathbf{w}}_{k}(n) \mathbf{M}^{\mathrm{H}}(n) \hat{\mathbf{C}}_{k}(n),
$$

and we have an alternative Lagrange equation with the iteration index $n$ :

$$
\mathcal{L}=\sum_{k=1}^{K} \sum_{f=1}^{F} A_{k f}^{2}(n+1)-\sum_{k=1}^{K} \lambda_{k}\left(\mathbf{g}_{k}(n) \mathbf{A}_{k}(n+1)-\varphi_{k}\right)
$$

Taking partial derivative of $\mathcal{L}$ with respect to $A_{k f}$ in equality to zero, it follows the solution under the constraint of (17) that

$$
A_{k f}(n+1)=\frac{\varphi_{k} g_{k f}(n)}{\sum_{f=1}^{F} g_{k f}^{2}}, \forall k, f .
$$

where $g_{k f}$ denotes the $f$ th element of $\boldsymbol{g}_{k}(n)$. Since $\tilde{\mathbf{w}}_{k}$ is a function of $\left\{A_{k f}\right\}$, the new $\left\{A_{k f}\right\}$ are used to update $\left\{g_{k f}\right\}$ for the next iteration. The derived $A_{k f}$ is generally a complex number, which means that both the amplitude and phase of each sub-carrier need to be adjusted in the transmitters to achieve the optimum condition. By this algorithm, sub-carrier selection for each user is simultaneously solved, where " $A_{k f}=$ 0 " means the $f$ th sub-carrier is not assigned to the virtual user $k$.

In implementation, this allocation algorithm is naturally realizable in centralized manner, where the power adjustments for all users are determined centrally according to the information of codes, channel status, MUD filter coefficients, and previously assigned powers. However, qusi-distributed operations could be also realizable since the iterative power update for each user needs not the information directly from others. It needs only the feedback of $\boldsymbol{g}_{k}$ for the virtual user $k$ and the allocation for every user is operated individually, although additional signaling overhead is potentially required.

\section{Numerical Simulations}

To demonstrate the allocation algorithm and the derived solutions, we simulate a system of two different data rates, where there are two basic-rate users (User 1, User 2) and two double-rate users (User 3, User 4), i.e., 6 virtual users, with SINR targets $(12 \mathrm{~dB}, 10 \mathrm{~dB}, 15 \mathrm{~dB}, 8 \mathrm{~dB})$ respectively. Random spreading codes ${ }^{1}$ are assigned and the basic spreading factor $F=16$ for either MC access or VSL access. For simplicity, we assume all path losses due to propagations to be unity without loss of generality and the sub-channels experienced by each user are uncorrelated Rayleigh fading with unit variance. The initial power allocation of each user is uniformly distributed to all sub-carriers as convention under $E_{b} / N_{0}=10 \mathrm{~dB}$, where $E_{b}$ denotes the energy per data bit.

\footnotetext{
${ }^{1}$ We use random spreading codes in simulations as general examples without loss of generality for other spreading codes
} 


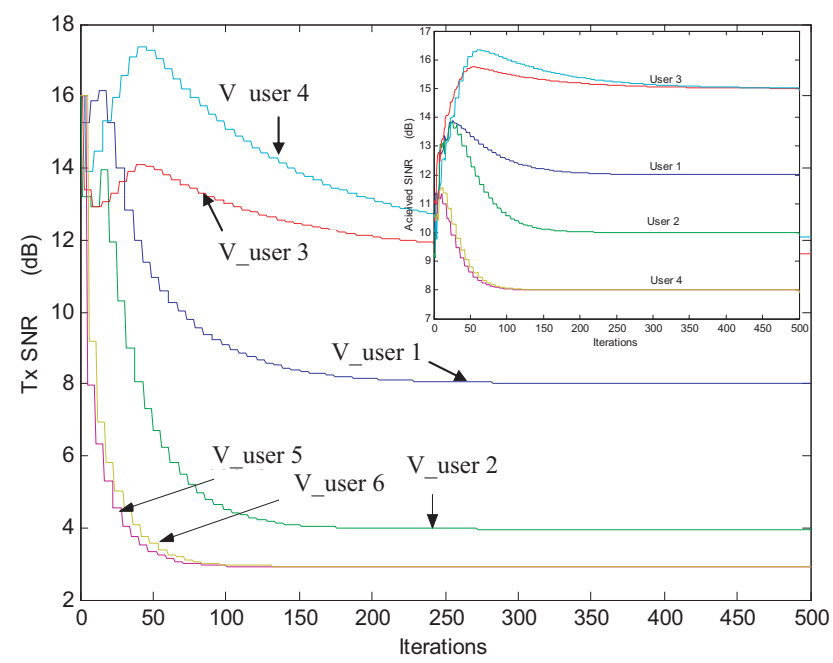

Fig. 3. The power update of user 1 (Virtual user 1), user 2 (Virtual user 2), user 3 (Virtual user $3 \& 4$ ), and user 4 (Virtual user $5 \& 6$ ) to achieve received SINR targets of $12 \mathrm{~dB}, 10 \mathrm{~dB}, 14 \mathrm{~dB}$, and $8 \mathrm{~dB}$ respectively in MC MC-CDMA, where $F=16$ under $E_{b} / N_{0}=10 \mathrm{~dB}$. The power is represented by $\mathrm{SNR}$ of the transmitted signal (Tx SNR).

Fig. 3 plots the power update curve of each virtual user in MC MC-CDMA with the achieved SINR approaching to their targets in the receiver (each curve represents one of the six virtual users). Fig. 4 shows the results in VSL MC-CDMA. Note that there should be 16 power update curves for each virtual user, where each curve corresponds to a sub-carrier, but we plot their power sum for not complicating the figures. The SINR of each virtual user is shown approaching to its target and we can find that the curves of the virtual users from the same user (we call them "homogenous virtual users") have similar behaviors in MC MC-CDMA but behave differently in VSL MC-CDMA. This is because the available sub-carriers for these homogenous virtual users are the same in MC access but not in VSL access, which is the result of their different strategies in realizing multi-rate access. Furthermore, it has the phenomenon of power concentration at several sub-carriers for each virtual user. For example, Fig. 5 specifically shows the 16 sub-carrier power-update curves of the two virtual users from User 4 in MC and VSL schemes respectively. The powers of the two virtual users concentrate on identical two sub-carriers in MC access but concentrate on different sub-carriers in VSL access.

To demonstrate a typical characteristic, Fig. 6 shows the power update curves in a MC MC-CDMA simulation run, where a random channel perturbation occurs, a user quits, and a user joins. We can see that in addition to converging to the steady solution, it fast adapts the channel changes and user variations. However, it is interesting that the power curves of the remaining virtual users do not change when the User 4 quits, and the steady solutions of these virtual users are identical to the previous after the participating of User 5. This phenomenon is actually the consequence of power concentration, discussed in the next section.

\section{Optimal Allocations}

According to the simulations, we observed that the power of each user in either MC MC-CDMA or VSL MC-CDMA

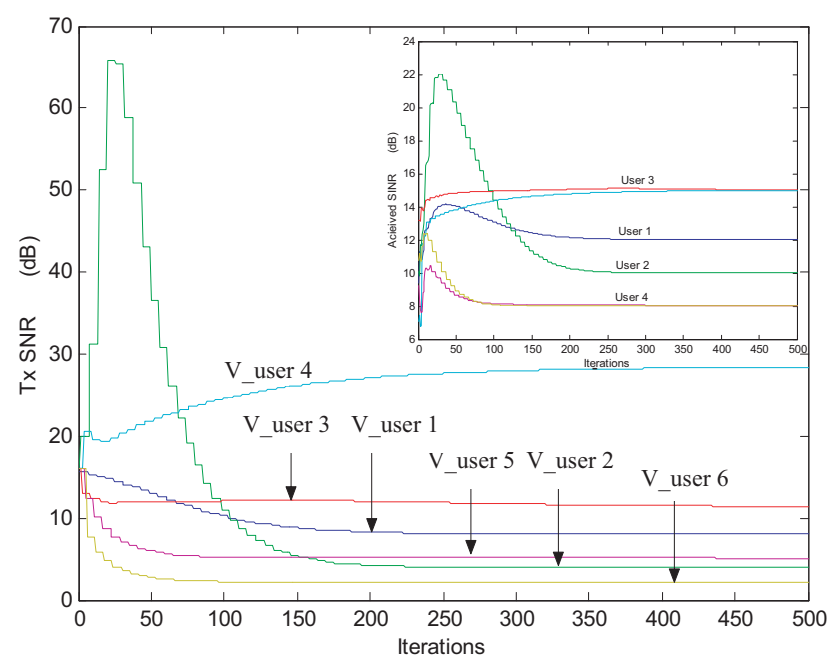

Fig. 4. The power update of user 1 (Virtual user 1), user 2 (Virtual user 2), user 3 (Virtual user $3 \& 4$ ), and user 4 (Virtual user $5 \& 6$ ) to achieve received SINR targets of $12 \mathrm{~dB}, 10 \mathrm{~dB}, 14 \mathrm{~dB}$, and $8 \mathrm{~dB}$ respectively in VSL MC-CDMA, where $F=16$ under $E_{b} / N_{0}=10 \mathrm{~dB}$. The power is represented by SNR of the transmitted signal (Tx SNR).

is concentrated on serval sub-channels whose maganitudes are relatively stronger with respect to each virtual user. If the basic spreading length of the system is larger than the number of active virtual users $(F \geq K)$ and every virtual user has a best sub-channel that is exclusive to others, the power of this virtual user is concentrated on such sub-carrier only, as shown in Fig. 5. It is equivalent to OFDMA that considers channel conditions and results in no co-channel interference among virtual users. It explains why in Fig. 6 the power curves of all remaining users keep unchanged after the quit of user 4.

On the other hand, if not every virtual user has an exclusively best sub-channel (e.g. in MC MC-CDMA) or $F<$ $K$, these 'colliding' virtual users share several stronger subchannels by spreading codes to achieve power minimization. Compared with OFDMA that always assigns sub-channels exclusively, sharing good sub-channels reflects the flexibility of MC-CDMA in sub-carrier selection and power allocations. The user capacity is not hard-limited by the available subcarriers but soft-limited by QoS demands. Regardless of channel coding schemes, MC-CDMA shows its advantage compared with pure orthogonal frequency multiple access in terms of radio resource utilization. For example, every OFDMA user can only exclusively select its own sub-carriers without the flexibility to share with others [9], [15], which is generally sub-optimal.

As a comparison, MC access is shown more power efficient than VSL access to satisfy identical rate and BER constraints because of its higher carrier-selection diversity in MC- CDMA, just like the example at Fig 5, unless all users are basic-rate, where MC and VSL are equivalent. Such difference in power consumption is shown in our plenty of simulations.

\section{CONCLUSION}

To achieve effective and quality-guaranteed wireless multimedia communication beyond $3 \mathrm{G}$, we proposed the resource allocation methods for MC-CDMA systems those adopt MC/VSL multi-rate access and LMMSE MUD receivers. We 


\section{MC-CDMA}
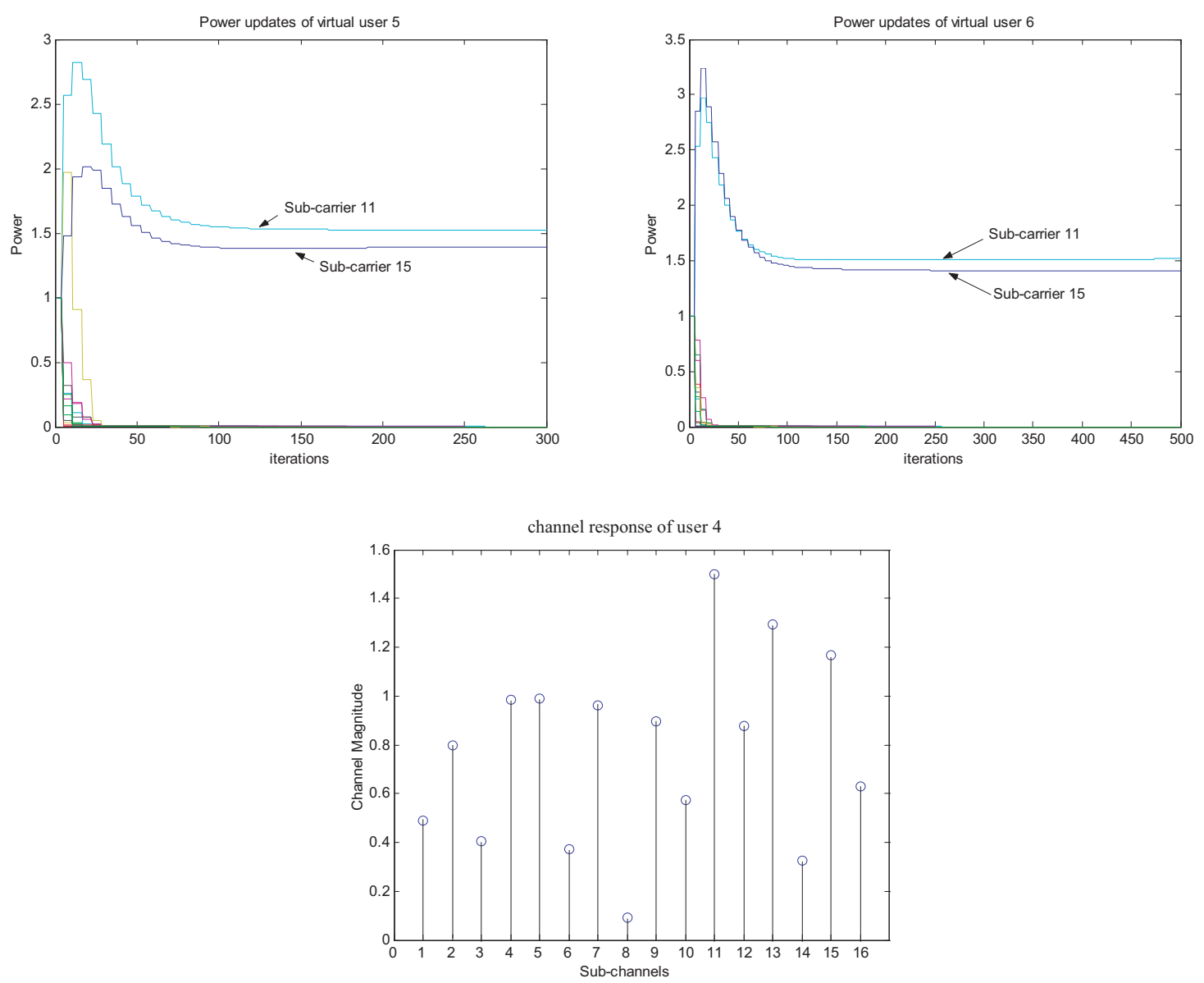

\section{VSL MC-CDMA}
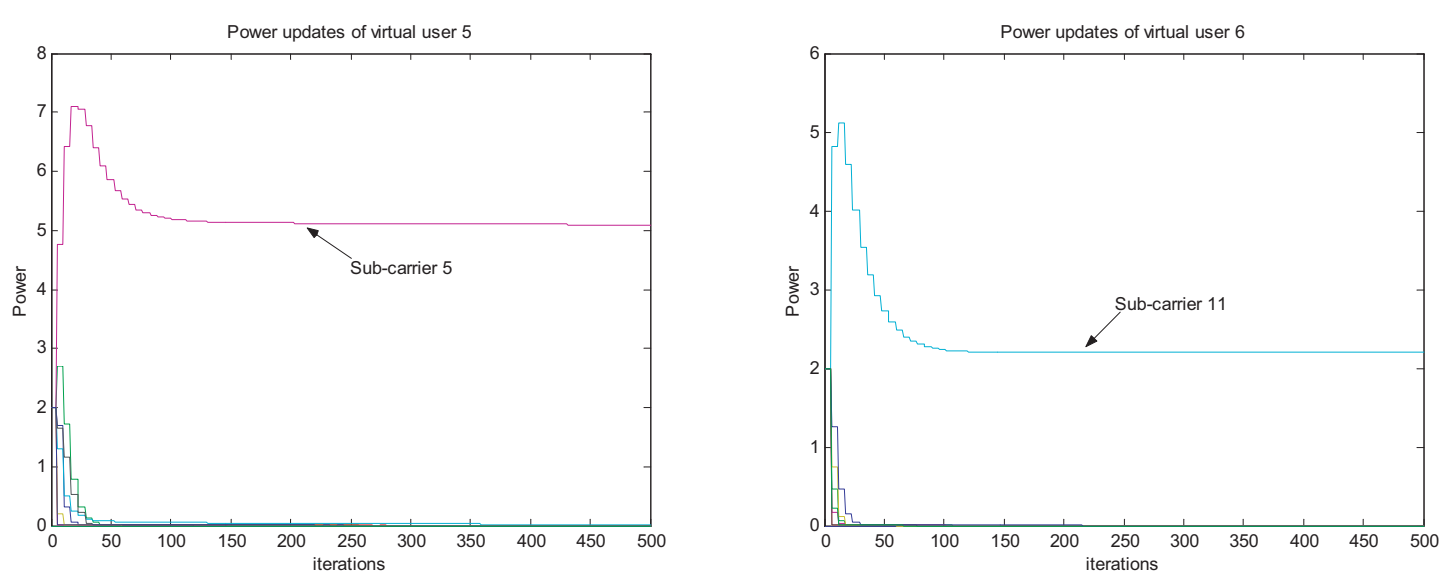

Fig. 5. Continued from the example of Fig. $3 \& 4$. The magnitude of channel response of User 4 and the sub-carrier power allocation for the corresponding V_User 5 \& V_User 6 in MC MC-CDMA and VSL MC-CDMA respectively. Note that V_user 5 can only use sub-carrier 1 to 8 and V_user 6 can only use sub-carrier 9 to 16 in VSL access.

determined the user capacity and derived a user (or service) admission criterion, which is a simple linear rule that relates the admissible rate, BER demands, and the number of subcarriers. It can be used not only to identify the supportable transmission rates of the system but also to decide the entrance of new users or new data streams. The proposed iterative algorithm jointly selects the sub-carriers and allocates the power for all users. Not limited to MC/VSL access, this algorithm is applicable to general MC-CDMA systems. The simulations also showed that when total transmitted power is minimized, the best-quality sub-carrier with respect to each virtual user is selected and power concentrated, which occurs when every virtual user has one exclusively best sub-channel. Otherwise, sub-carriers of better quality are shared among virtual users by spreading codes. 


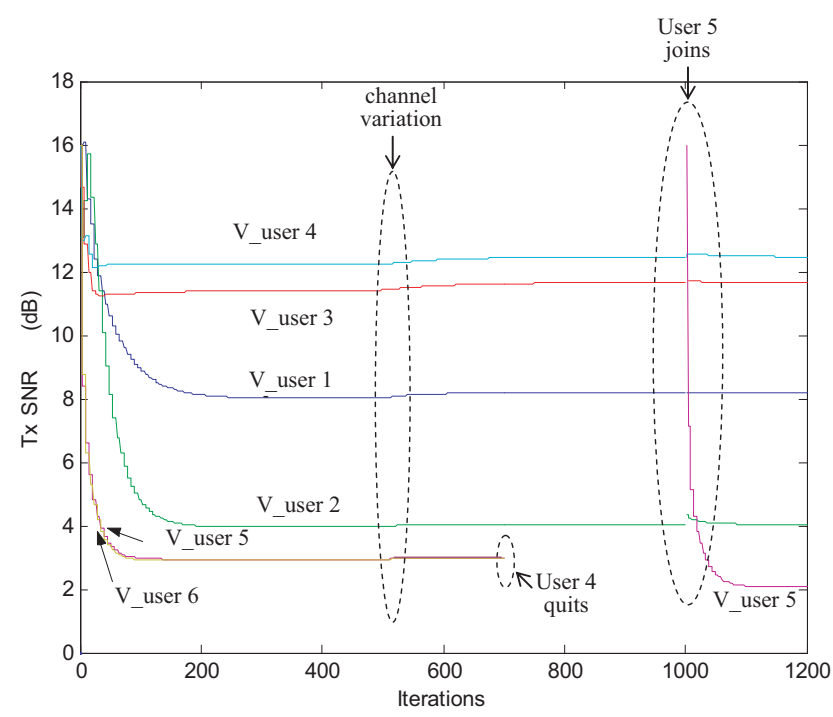

Fig. 6. Transmit power adjustments of each virtual user in a MC MCCDMA example. V_user 1 and 2 are from the basic rate User 1 and User 2 respectively. V_user 3 and V_user 4 are the virtual users from user 3; V_user 5 and V_user 6 are the virtual users from user 4 . The virtual user from the new joined basic-rate user 5 is number as " 5 " after the quit of user 4 .

\section{REFERENCES}

[1] A. Chouly, A. Brajal, and S. Jourdan, "Orthogonal multicarrier techniques applied to direct sequence spread spectrum CDMA systems," in Proc. of IEEE Global Com. Conference, 1993, pp. 1723-1728.

[2] N. Yee, J. P. Linnartz, and G. Fettwies, "Multi-carrier CDMA in indoor wireless radio networks," in Proc. of IEEE Personal, Indoor and Mobile Radio Communications Conference, 1993, pp. 109-113.
[3] S. Hara and R. Prasad, "Overview of multicarrier CDMA," IEEE Comm. Magazine, pp. 126-133, Dec 1997.

[4] Y. Zhu and E. Gunawan, "Performance of MC-CDMA system using controlled MRC with power control in Rayleigh fading channel," IEEE Electronics Letters, pp. 752-753, Apr. 2000.

[5] L. Jorguseski, E. Fledderus, J. Farserotu, and R. Prasad, "Radio resource allocation in third generation mobile communication systems," IEEE Commun. Mag., pp. 117-123, 2001.

[6] S. W. Kim and Y. H. Lee, "Combined rate and power adaptation in DSCDMA communications over Nakagami fading channels," IEEE Trans. Commun., pp. 162-168, January 2000.

[7] J. B. Kim and M. Honig, "Resource allocation for multiple classes of DS-CDMA traffic," IEEE Trans. Vehic. Technol., pp. 506-519, March 2000.

[8] S. Moshavi, "Multi-user detection for DS-CDMA communications," IEEE Commun. Mag., pp. 124-136, Oct. 1996.

[9] R. V. Nee and R. Prasad, OFDM for Wireless Multimedia Communications. Artech House, 2000.

[10] T. Ottosson and A. Svensson, "Multi-rate schemes in DS/CDMA systems," in Proc. IEEE Vehic. Tech. Conf., 1995, pp. 1006-1010.

[11] P. W. Fu and K. C. Chen, "Multi-rate multi-carrier-CDMA with multiuser detections for wireless multimedia communications," in Proc. of IEEE WCNC 03, Mar. 2003, pp. 385-390.

[12] H. V. Poor and S. Verdú, "Probability of error in MMSE multiuser detection," IEEE Trans. Info. Theory, pp. 858-871, May 1997.

[13] U. Madhow and M. L. Honing, "MMSE interference suppression for direct-sequence spread-spectrum CDMA," IEEE Trans. Commun., pp. 3178-3188, Dec. 1994.

[14] T. M. Lok and T. F. Wong, "Transmitter and receiver optimization in multicarrier CDMA systems," IEEE Trans. Commun., pp. 1197-1207, July 2000.

[15] C. Y. Wong et al., "Multiuser OFDM with adaptive subcarrier, bit, and power allocation," IEEE J. Select. Areas Commun., pp. 1747-1758, Oct. 1999.

[16] D. P. Bertsekas, Constrained Optimization and Lagrange Multiplier Methods. Academic Press, Oct. 1982. 